\title{
Estimating bradykinesia severity in Parkinson's disease by analysing gait through a waist-worn sensor
}

\author{
A.Samà* 1,3, C.Pérez-López ${ }^{* 1,3}$, D.Rodríguez-Martín' ${ }^{1}$, A.Català1,3 J.M. Moreno-Aróstegui 1,3, \\ J. Cabestany ${ }^{1,3}$, E. de Mingo², A. Rodríguez-Molinero ${ }^{2,3}$ \\ ${ }^{1}$ Technical Research Centre for the Dependency Care and Autonomous Living (CETpD), \\ Universitat Politècnica de Catalunya - BarcelonaTech (UPC), Spain \\ \{albert.sama, carlos.perez-lopez, daniel.rodriguez-martin, andreu.catala, joan.manuel.moreno, joan.cabestany\}@upc.edu \\ ${ }^{2}$ Clinical Research Unit, Consorci Sanitari del Garraf (Fundació Privada Sant Antoni Abat), Spain \\ rodriguez.molinero@gmail.com, itzahe1@hotmail.com \\ ${ }^{3}$ Sense4Care, Spain \\ * These authors contributed equally
}

\begin{abstract}
Bradykinesia is a cardinal symptom of Parkinson's disease (PD) and describes the slowness of movement revealed in patients. Current PD therapies are based on dopamine replacement, and given that bradykinesia is the symptom that best correlates with the dopaminergic deficiency, the knowledge of its fluctuations may be useful in the diagnosis, treatment and better understanding of the disease progression. This paper evaluates a machine learning method that analyses the signals provided by a triaxial accelerometer placed on the waist of PD patients in order to automatically assess bradykinetic gait unobtrusively. This method employs Support Vector Machines to determine those parts of the signals corresponding to gait. The frequency content of strides is then used to determine bradykinetic walking bouts and to estimate bradykinesia severity based on an epsilon-Support Vector Regression model. The method is validated in 12 PD patients, which leads to two main conclusions. Firstly, the frequency content of the strides allows for the dichotomic detection of bradykinesia with an accuracy higher than $90 \%$. This process requires the use of a patient-dependant threshold that is estimated based on a leave-one-patient-out regression model. Secondly, bradykinesia severity measured through UPDRS scores is approximated by means of a regression model with errors below $10 \%$. Although the method has to be further validated in more patients, results obtained suggest that the presented approach can be successfully used to rate bradykinesia in the daily life of PD patients unobtrusively.
\end{abstract}

Keywords: Support Vector Machines, Inertial sensors, Bradykinesia, Parkinson's disease 


\section{Introduction}

. Parkinson's disease (PD) pathology is typified by the death of the dopamine-producing neurones, being dopamine a neurotransmitter required for a correct movement control [1]. The first noticeable PD signs correspond to an affected regulation of movement, and they are due to the said lack of dopamine-producing cells. This way, current PD treatments are based on increasing dopamine levels, being levodopa the most extended one. Although this active ingredient temporally reverts the symptoms, it does not prevent disease progression. Bradykinesia is one of the most incapacitating PD motor symptoms, and it is used to describe the pathological slowness of movement [2]. In addition to this, it is the main symptom related to basal ganglia disorders and, although it is a cardinal symptom of PD, it might also be present in other disorders, e.g. depression [1]. Bradykinesia is sometimes also used to describe two other motor disorders: akinesia and hypokinesia. Akinesia is related with a poverty of spontaneous movement, while hypokinesia describes decreased bodily movements [1]. In this article, we use bradykinesia to refer to those difficulties with planning, initiating and executing movement and with performing sequential and simultaneous tasks $[1,2]$. Although the pathophysiology of bradykinesia is not well known, it has been shown that, in patients with PD, bradykinesia is the symptom that best correlates with dopaminergic deficiency [3].

In current clinical practice, the assessment of bradykinesia includes the execution of rapid, repetitive, alternating movements of the hand and heel tapping while the clinician observes the slowness and amplitude of the movements [1]. This kind of assessment is employed in the Unified Parkinson's Disease Rating Scale (UPDRS), which is a clinical tool widely utilised by neurologists to evaluate different aspects of PD. UPDRS is a questionnaire divided into 4 parts: non-motor aspects, motor aspects, motor examination and motor complications; each part comprises several items that are rated with a score between 0 and 4. The third part of the UPDRS assesses the motor signs of PD as a patient manifests them at the moment of the assessment, being bradykinesia evaluated through several items in this third part. However, as explained above, bradykinesia is a symptom that can appear and disappear throughout the day and its presence and severity vary, among others, with the moment and quantity of the last medication intake and the punctual absorption response of the patient. Furthermore, the severity of this symptom also depends on the emotional state of the patient and the environment [1]. Thus, given that UPDRS is a punctual assessment, it does not show, in general, the real severity of the bradykinesia. In addition to 
this, the administration of UPDRS is very time-consuming for therapists, and the repeated administration of the scale is frustrating for patients since they have to repeat specific movements several times to re-evaluate their condition. Thus, the use of the UPDRS items in the evaluation of bradykinesia is burdensome and may provide biased information from the true scope of the symptom.

Novel signal processing methods and wearable devices have been recently developed to assess specific items of the UPDRS automatically, therefore enabling its evaluation at patients' home as in $[4,5]$. However, their usage is restricted to few times a day, since they require patients to perform specific exercises. In consequence, a system able to assess the onset of bradykinesia and its severity during patients' activities of daily living (ADL), without requiring to perform specific exercises, would be of great help in clinical practice. Given the correlation between bradykinesia and dopamine levels, the use of such systems would enable doctors to enhance the tailoring of the medication intakes and, therefore, improve the response to treatment. Additionally, the knowledge of the bradykinesia evolution may be a good indicator of the disease advance for neurologists. Finally, in patients with continuous infusion pump treatments, determining bradykinetic periods in real-time could open the possibility to automatically administrate rescue doses or bolus in order to avoid OFF periods.

This work presents a new machine learning method to assess and quantify bradykinesia by means of a single waist-worn accelerometer. The method is based on a Support Vector Machine (SVM) classifier that detects gait, a specific signal processing method that detects strides and, finally, a characterisation of these strides based on their frequency content. The resulting frequency characterisation is then entered into a regression method to estimate bradykinesia severity in terms of UPDRS scores. The device and method were tested with signals recorded from 12 PD patients while performing a set of scripted ADL at home. The method presented in this paper shows that an accurate monitoring of bradykinesia can be obtained from patients' gait through a single waist-worn device, with an average accuracy above $90 \%$. Furthermore, results show that the method's output is highly correlated with UPDRS scores (correlation coefficient $r>0.9$ ). Finally, bradykinesia-related items of the UPDRS are approximated by an epsilonSupport Vector Regression ( $\varepsilon-$ SVR) providing errors below $10 \%$ in some cases.

The paper is organised as follows. The next section presents several studies in which bradykinesia was assessed based on wearable sensors. Section 3 is devoted to describing the signal processing and machine learning approach to analyse bradykinesia. Section 4 describes the data collection with PD patients and the data analysis. Finally, results, discussion, and conclusions are detailed. 


\section{Related Work}

The research conducted so far on the detection of bradykinesia by means of inertial sensors is mainly based on characterising patients' movements, which was followed by one of the first works conducted in this field by Dunnewold et al. [6]. This study used ambulatory monitoring to quantify bradykinesia and hypokinesia in a population of 50 PD patients. To this end, two wrist-worn accelerometers were used. Results demonstrated limited sensitivity, around $60-71 \%$ and specificity of $66-76 \%$ in individual PD patients. Furthermore, the objective measures of bradykinesia in this study did not show any relationship with the score of the UPDRS.

Researchers at the École Polytechnique Fédérale de Lausanne evaluated the use 7 gyroscopes and 2 accelerometers located on the forearms, shins, and trunk to represent the presence or absence of tremor, bradykinesia, postural transitions, body posture and gait parameters [7]. The results showed correlations up to 0.71 with the bradykinesia UPDRS scores. Following this work, Salarian et al. used a wrist sensor to detect tremor and extract parameters related to bradykinesia in 20 PD patients [8]. Bradykinesia was measured in periods during which the patient moved the upper extremities. The estimated values were compared to the summation of specific UPDRS items, while Pearson's correlation was used between UPDRS subscores and the 3 parameters, showing values between -0.42 to -0.76 .

Zwartjes et al. assessed bradykinesia and hypokinesia in PD patients while they were asked to perform certain daily tasks and UPDRS motor tests in a randomly predefined order [9]. Motor activity was measured using four inertial sensors placed on the trunk and wrist, thigh and foot of the most affected side of patients. Bradykinesia was characterised by the average value of acceleration, step length and step velocity, and other parameters. Hypokinesia parameters were characterised as how patients moved their arms. As these parameters cannot directly be translated into single UPDRS items, authors chose to compare them to the item that represents the overall bradykinesia and hypokinesia. None of the hypokinesia-related parameters was significantly correlated with this UPDRS item.

Recently, a study originated from a European research project called PERFORM was published by Cancela et al. [10]. In this paper, the authors presented a motor symptom monitoring system that was evaluated on twenty patients performing a scripted set of ADL. Several classification algorithms were tested, being SVM the one with the highest accuracy. In their paper, the algorithms assessed both the 
presence and severity of bradykinesia. Later, a modified version this algorithm was developed on the basis of inertial signals collected from 24 patients performing unscripted activities at their homes [11]. Results showed an accuracy of $74.4 \pm 14.9 \%$ in detecting bradykinesia UPDRS scores. Nevertheless, in both cases, the system is composed of a set of five wearable sensors and a central store unit making the system unusable as a continuous monitoring for assessing ADL.

A wrist-worn sensor called Kinetigraph has also been evaluated in the automatic assessment of bradykinesia [12]. This device employs a triaxial accelerometer and analyses the frequency content within the bands of 0.2 to $4 \mathrm{~Hz}$ in order to produce bradykinesia scores every two minutes. These scores were compared to UPDRS part III rating values. The correlation coefficient among them was 0.64 . The only error measurement that we could find in this paper states that Kinetigraph bradykinesia scores provided a margin of error of 18 UPDRS III units.

A distinct tendency of the evaluation of bradykinesia in PD involves scoring patient's response to some exercises, similarly to the evaluation of the UPDRS, based on the signals provided by specific sensors. For instance, Kim et al. [13] used a gyroscope to characterise the velocity and amplitude of finger tapping exercises. These characteristics show a correlation of 0.75 with UPDRS scores related to the tap test. In the same way, Houde Dai el al. [14], in a recent article published in 2015 in which nine PD patients participated, obtained correlations up to 0.83 between the UPDRS bradykinesia score and measurements extracted from the finger tap test. Similarly, Kinesia device, which was developed by Great Lakes Neurotechnology, consists of a triaxial accelerometer and a gyroscope which analyses the movement of patient's finger [15]. Finally, eight sensors were employed by researchers from Harvard Medical School to estimate UPDRS score during scripted movement exercises [5]. These methods have the disadvantage of requiring patients to wear several sensors, which might be cumbersome to patients and, furthermore, to perform specific exercises, other than those usually performed during daily life. In consequence, fluctuations may be monitored discontinuously, and rapid fluctuations may be lost. In addition, these types of devices require patients to stop doing their regular activities.

\section{Unobtrusive bradykinesia assessment method}

This section presents the approach followed to determine, in an unobtrusive way, the presence of bradykinesia and, furthermore, an estimation of its severity. This approach is applied to the signals obtained 
by a tri-axial accelerometer. As previously described, the accurate knowledge of bradykinesia and its severity along a day may be useful for the diagnosis and better understanding of the disease progression. However, bradykinesia assessment should be given to clinicians in a standardised way. In consequence, UPDRS ratings are considered as the measurement for bradykinesia severity.

The rationale behind the method relies on the fact that motor activities are a sequence of movements that have different degrees of automation and, additionally, these automatic movements are slower in PD patients due to an increase of start-up time and a slow implementation of the movement [37]. Thus, since the most characteristic and repetitive automatic human movement is gait and it has a high degree of automation, gait is considered to be the most suitable activity to objectively analyse bradykinesia, understood as the absence, slowness and progressive reduction of movement. This way, an automatic assessment of bradykinesia without requiring the patient to perform any specific movement could be achieved. Although this method assesses bradykinesia when patients walk, it is capable of providing information several times a day since patients, even in moderate or advanced phases of the disease, walk more than 40 times per day [16,17]. This way, this method is capable of assessing the onset of bradykinesia and its severity during patients' ADL during many occasions a day.

In order to unobtrusively assess bradykinesia, a tri-axial accelerometer has been located at patient's waist since this position had been previously considered as one of the most comfortable ones [18] and, in addition to this, it ensures to cover most of the movements obtained during gait due to its closeness to the centre of mass of the human body. Furthermore, this approach allows the monitoring of other PD symptoms such as dyskinesia [19]. Figure 1 shows the position in which the sensor is worn. A neoprene belt is used to attach it to the lateral side of the waist. 


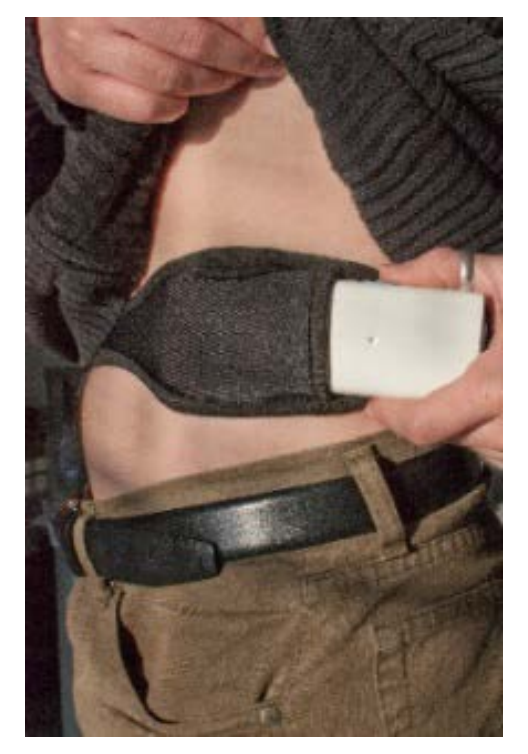

Figure 1: Wearable sensor and neoprene belt used to attach it to the lateral side of the waist

Figure 2 shows a block diagram that outlines the bradykinesia assessment method that is applied to the acceleration signals. The method is divided into two main parts: characterisation of gait and quantification of bradykinesia, which are detailed in the following subsections. The first part of this method (gait characterisation) has been previously presented in [19] and [20]. Gait is characterised by firstly detecting when a patient is walking through an SVM model. Secondly, strides are identified and, finally, they are characterised through a frequency feature. Then, given a set of strides characterised by this frequency feature, the presence of bradykinesia is determined by comparing a threshold $\beta$ with the average of the frequency features. This threshold $\beta$ is obtained through an $\varepsilon-S V R$ model that is built through a leave-one-patient-out approach. In addition to the detection of the symptom, bradykinesia severity is estimated by entering the frequency features from the gait characterisation into a second $\varepsilon$-SVR model, in which some characteristics of patients' disease stage are also provided. This model estimates bradykinesia severity as UPDRS's third-part items. 


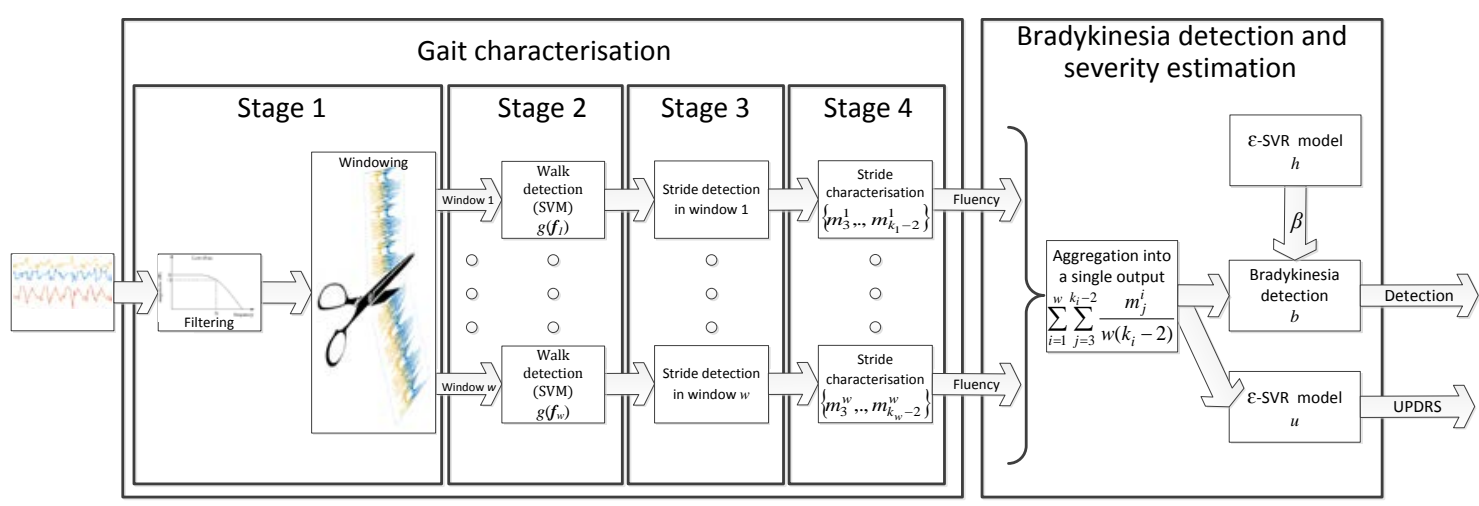

Figure 2: Bradykinesia detection and severity estimation

\subsection{Gait characterisation}

As previously described, gait is analysed in order to characterise and assess bradykinesia. Currently, the employed method, in which clinicians assess gait bradykinesia, consists in observing the fluidity of patients' movements during gait. In consequence, the main feature used to characterise the symptoms is motion fluency while patients are walking.

The first stage, signal pre-processing, consists of the conditioning and windowing of the accelerometer samples. Signal conditioning is performed through a second-order low-pass Butterworth filter with a cut-off frequency of $15 \mathrm{~Hz}$. Then, the filtered signals are segmented into overlapped windows of $l$ seconds, each window starting every $l / 2$ seconds. As a result, a certain window $w$ contains $l \cdot F_{s}$ samples from each of the three axes of the accelerometer, where $F_{s}$ is the sampling frequency, and windows start every $l \cdot F_{s} / 2$ samples.

Once the signal is windowed, gait detection is performed on each window separately. This is handled as a supervised learning problem. With this purpose, SVM are chosen given that the problem is to classify into two classes: provided the signals comprised in a certain time window $w$, we need to determine whether the patient is walking or not. To this end, signals within a window are characterised by a set of $k$ features, i.e. a window $w$ is represented by its feature vector $\boldsymbol{f}_{w}=\left[f_{1}^{w}, \ldots, f_{k}^{w}\right]$, where $f_{j}^{w}$ is the $j^{\text {th }}$ feature of window $w$. Thus, given a training set of feature-vector representation of several windows $\left\{\boldsymbol{f}_{1}, \ldots, \boldsymbol{f}_{n}\right\}$ and its corresponding labels $\left\{y_{1}, \ldots, y_{n}\right\}$ where $y_{w}=\{1,-1\}$, being 1 if the patient walked and -1 otherwise, the following minimisation problem is solved in order to obtain a gait detection classifier: 


$$
\begin{gathered}
\min _{w, b, \xi} \frac{1}{2}\|\boldsymbol{w}\|_{2}^{2}+C \sum_{i=1}^{m} \xi_{i} \\
\text { s.t } \xi_{i} \geq 0 \\
y_{i}\left[K\left(\boldsymbol{w}, \boldsymbol{f}_{i}\right)+b\right] \geq 1-\xi_{i}
\end{gathered}
$$

where $K\left(\boldsymbol{w}, \boldsymbol{f}_{i}\right)=e^{\gamma\left\|\boldsymbol{w}-\boldsymbol{p}_{i}\right\|_{2}^{2}}$ is the Radial Basis Function kernel, $b$ is the hyperplane bias, $\boldsymbol{w}$ is the hyperplane that separates both classes and $\xi_{i}$ are the slack variables.

In a previous study [19], 800 frequency features consisting of the energy of the signal in 800 frequency bands computed from signals collected from 10 patients, were analysed. The most suitable features to detect walk were identified by the Relief algorithm as those that maximised inter-class distance and minimised the intra-class one [6]. As a result, two features $(k=2)$ were identified and used to detect walking, consisting in the energy contained within the frequency bands $[0.1,3] \mathrm{Hz}$ and $[0.1,10] \mathrm{Hz}$. These features are noted as $f_{1}^{w}$ and $f_{2}^{w}$, respectively. The SVM model was then found by optimising values for $C$ and $\gamma$ through a 10-fold Cross Validation and using data of the same 10 PD patients.

Based on the same study [19], the number of samples comprised in a window was set to $l=128$ with the aim of enabling an efficient computation of the Discrete Fourier Transform based on Fast Fourier Transform [21]. In addition to this, the sampling frequency was fixed to $F_{s}=40 \mathrm{~Hz}$, since frequency content of gait is below $20 \mathrm{~Hz}$ [22], thus providing a window duration $w$ of 3.2 seconds. In order to ensure that all events are captured, a new window is started every $l / 2$ samples.

The label of a new window represented by its feature vector $\boldsymbol{f}$ is then obtained by:

$$
g(\boldsymbol{f})=\operatorname{sgn}\left(\sum_{i=1}^{m} y_{i} \alpha_{i} K\left(\boldsymbol{f}, \boldsymbol{f}_{i}\right)+b\right)
$$

where the set of $\alpha_{i}$ are the Lagrangian multipliers of the SVM dual problem formulation.

After detecting that a patient is walking, strides are detected in terms of walking bouts. A walking bout is defined as a collection of consecutive windows in which gait is detected, i.e. $g\left(\boldsymbol{f}_{x+1}\right)=\cdots=$ $g\left(\boldsymbol{f}_{x+U}\right)=1$. This way, given a walking bout, steps and strides are detected within the acceleration samples contained in these $U$ windows. To this end, the principles described in Zijlstra's work have been employed [23]. More specifically, the initial contact event is identified as the local minimum in the frontal acceleration measured from the waist [24]. This local minimum reflects the deceleration sensed at the initial contact and it is considered to be the beginning of a step. In our sensor location (left-side of the 
waist), this local minimum is better observed when the step is done by the left leg; but local minima are still observed in the steps from the opposite side. Nonetheless, our interest focuses on strides [1]. In this case, left and right steps are distinguished by analysing the lateral acceleration, since its period is twice the frontal one during gait [23]. In consequence, relative extrema are detected to identify left and right steps comprising strides. This way, signals in those windows, in which the SVM model detects gait, are segmented into strides.

More formally, the strides detected in the $n^{\text {th }}$ walking bout are represented by a set $\left\{S_{1}^{n}, \ldots, S_{k_{n}}^{n}\right\}$, where each $S_{i}^{n}$ is a three-column matrix containing the stride acceleration measurements. Each stride starts at time $\left\{t_{1}^{n}, \ldots, t_{k_{n}}^{n}\right\}$. However, not all strides in a walking bout are analysed. The first and last two strides are excluded from the analysis because we aim to analyse bradykinesia as an automatic movement and automatic activities enable patients to manifest the symptom. In consequence, strides corresponding to gait initiation and gait termination are excluded. More formally, the subset of strides $\left\{S_{3}^{n}, \ldots, S_{k_{n}-2}^{n}\right\}$ is analysed, so that only those walking bouts with $k_{n}=5$ or more strides are used.

Gait cycles $S_{i}^{n}$ are then individually characterised with the aim of representing the presence of bradykinesia. The basis of this characterisation is the previously mentioned study [19]. In this study, several features were tested in 20 PD patients. These features were analysed by their ability to linearly separate the presence of the symptom (together with motor states) and, also, they were found to intuitively represent the fluidity of movement. From the conclusions of this work, the best feature to characterise the fluidity of movement was the energy in the $(0,10] \mathrm{Hz}$ frequency bands of the stride. Finally, given the set of stride detected on the accelerometer signal during the $n^{\text {th }}$ walking bout, represented by $\left\{S_{3}^{n} \ldots, S_{k_{n}-2}^{n}\right\}$, their $(0,10] \mathrm{Hz}$ motion fluency feature is noted as $\left\{m_{3}^{n}, \ldots, m_{k_{n}-2}^{n}\right\}$.

This fluency feature has been previously tested to estimate the ON/OFF motor states of 15 PD patients in a previous work [20]. However, this feature has not been analysed in the task of detecting bradykinesia and estimating its severity, which is the main purpose of this work.

\subsection{Bradykinesia detection}

Bradykinesia detection is performed based on the motion fluency values from several strides $\left\{m_{i}^{n}\right\}$. These fluency values, corresponding to the energy in specific frequency bands of the strides, provide a higher value with more fluent strides. Similarly, less fluent strides provide lower $m_{i}^{n}$ values. In consequence, 
we consider a certain threshold to classify fluency values into bradykinetic and non-bradykinetic strides.

More formally, let $\beta$ be a scalar value; then, if $m_{i}^{n} \leq \beta$, it is inferred that the patient presented bradykinesia during the stride $i$ of the $n^{\text {th }}$ walking bout and, on the contrary, bradykinesia is inferred to be absent if $m_{i}^{n}>\beta$. However, we avoid using individual strides since bradykinesia is a symptom that commonly lasts for several minutes. Hence, strides from walking bouts are considered together in order to obtain a more robust assessment of bradykinesia, as in [19]. This way, bradykinesia detection is considered on each walking bout according to:

$$
b^{n}=\left\{\begin{array}{ccc}
\text { Bradykinesia } & \text { if } & \sum_{i=3}^{k_{n}-2} \frac{m_{i}^{n}}{k_{n}-4} \leq \beta \\
\text { No bradykinesia } & \text { if } & \sum_{i=3}^{k_{n}-2} \frac{m_{i}^{n}}{k_{n}-4}>\beta,
\end{array}\right.
$$

Bradykinesia detection relies on threshold $\beta$ that determines the minimum fluidity to consider that bradykinesia is not present. This threshold is different among patients, since each patient's gait is differently affected by the disease and, thus, each patient walks in a different way. Therefore, $\beta$ must be personalised to each patient.

\subsection{Bradykinesia detection adjustment}

The adjustment of $\beta$ to each patient is presented as a regression problem. We want to find a function $h: \mathbb{R}^{d} \rightarrow \mathbb{R}$ that, given some characteristics from a patient represented as a $d$-dimensional real vector, provides the personalised scalar value $\beta$.

The adjustment of $\beta$ to each patient is done according to the following observations. Given that bradykinesia is a symptom that fluctuates and it is correlated to the dopamine level:

- During low dopamine-level periods, patients show the bradykinesia severity that corresponds to their disease stage. Disease stage is commonly measured through the Hoehn \& Yahr (H\&Y) scale, which measures the progress of symptoms through a rating scale with a mark between 1 (initial stage of the disease) and 5 (advanced stage). In consequence, patients with higher H\&Y will manifest more severe bradykinesia and lower $m_{i}^{n}$ fluidity values during low dopaminelevel periods. 
- During normal dopamine-level periods, patients do not present bradykinesia; their movement is not slowed-down by the disease, and, therefore, it is fluid. In addition, age is a factor that has shown to limit movement fluidity; hence the older a patient is, the less fluid the movement can be. In consequence, we expect higher $m_{i}^{n}$ during these periods, but they are limited by patients' age.

Based on these observations, the $\beta$ threshold is considered to depend on the H\&Y (higher H\&Y provide lower $\beta$ ) and the age (older patients would also present lower $\beta$ ) from each patient.

Furthermore, patients' movement fluidity is considered to be unique to each patient, since the severity with which other symptoms (e.g. freezing of gait) are presented in each patient is also unique. In consequence, the range of values that fluidity measurements $\left\{m_{i}^{n}\right\}$ may cover is different in each patient and it should be taken into account to adjust $\beta$, as well as statistical parameters that define its distribution. This way, minimum, maximum, mean, median and standard deviation values are considered to be relevant in adjusting $\beta$.

Provided these observations, the information from a certain patient $i$ is characterised through a 7dimensional vector defined by:

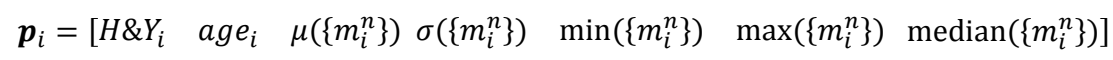

where $\mu(\cdot)$ corresponds to the mean value, and $\sigma(\cdot)$ to the standard deviation.

Finally, the mapping $h: \mathbb{R}^{d} \rightarrow \mathbb{R}$ that provides $\beta$ is implemented as a regression function. The process to obtain mapping $h$ is depicted in Figure 3. This regression is learned through, firstly, a set of $\left\{\boldsymbol{p}_{i}\right\}$ and the optimal $\left\{\hat{\beta}_{i}\right\}$ from each patient. $\hat{\beta}_{i}$ is defined as the value of $\beta$ that maximises the specificity and sensitivity in detecting bradykinesia for patient $i$. As Section 4.3 describes, optimal thresholds $\left\{\hat{\beta}_{i}\right\}$ are obtained for each patient through data analysis; furthermore, these data are employed by using a leaveone-patient-out strategy in order to avoid overfitting and ensuring that the method is implementable for any new patient. Secondly, mapping $h$ is modelled by means of an $\epsilon-\operatorname{SVR}[25,26]$ model with RBF kernel. This type of regression locates a tube to estimate the output variable with an $\varepsilon$-insensitive loss function as follows: 


$$
\begin{aligned}
\min _{w, b, \xi \xi \xi *} \frac{1}{2}\|\boldsymbol{w}\|_{2}^{2}+C \sum_{i=1}^{m}\left(\xi_{i}+\xi_{i}^{*}\right) \\
\text { s.t. } \quad \hat{\beta}_{i}-\left[w \cdot x_{i}+b\right] \leq \varepsilon+\xi_{i} \\
{\left[w \cdot x_{i}+b\right]-\hat{\beta}_{i} \leq \varepsilon+\xi_{i}^{*} } \\
\xi_{i}, \xi_{i}^{*} \geq 0
\end{aligned}
$$

where $\xi_{i}^{*}$ are a second set of slack variables to cope with infeasible constraints of the optimisation problem. In this kind of regression, $\varepsilon$ defines the minimum deviation from the threshold, due to the insensitive loss defined by the equation $[25,26]$.

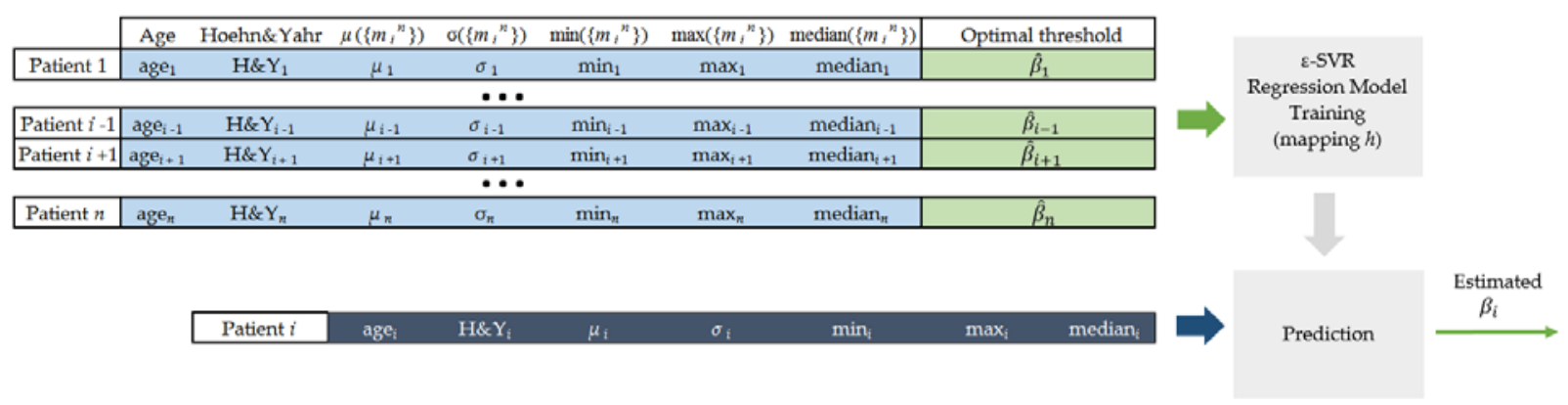

Figure 4: Estimation of the $\beta$ threshold for the $i^{\text {th }}$ patient by using age, HoehnEYahr and statistical values of the fluidity measurements. Note that only the optimal values $\hat{\beta}$ from the other patients are used to train the regression model, according to the leave-one-patient-out approach. Blue rows correspond to $\boldsymbol{p}_{j}$ vectors. The estimated $\beta_{i}$ value is used to predict if walking bouts from the $i^{\text {th }}$ patient are bradykinetic according to Equation (3). This process is independently applied to each patient in order to obtain their corresponding thresholds $\beta$.

\subsection{Bradykinesia severity estimation based on fluidity values}

As previously shown, UPDRS is employed in current clinical practice to evaluate several aspects of the disease. In its third part (motor examination), the motor state of a patient is punctually assessed. UPDRS is not only the current gold-standard tool for the evaluation of motor symptoms in PD, but it is also the most extended way for clinicians to define the severity of each symptom. Health professionals score each item included in the scale with a value between 0 and 4 . Among these items, several of them are directly related to bradykinesia, such as item 24, that rates 'Bradykinesia and hypokinesia', or item 22 for 'Gait'. 
In this paper, we propose to estimate bradykinesia severity as an automatic assessment of selected UPDRS items (described in Section 4), based on the previously described gait characterisation, as a tool to provide relevant information to the health professionals.

The problem at hand can be formulated as finding out a mapping $z$ that provides UPDRS scores:

$$
z: \mathbb{R}^{d} \rightarrow \mathbb{R} \in[0,4]
$$

Similarly to mapping $h$, patients' data are characterised as $d$-dimensional vectors. The same observations related to dopamine levels described in the previous section are applicable to the task of estimating UPDRS. In consequence, most important information from patients are also characterised as $\boldsymbol{p}_{i}$ (see Equation 4) according to their H\&Y, age and statistics of the fluidity values. Mapping $z$ is also implemented with an $\varepsilon-\operatorname{SVR}[25,26]$.

\section{Experiments}

\subsection{Participants and materials}

In this experiment, a total of 12 PD patients with idiopathic PD according to UK PD Society Brain Bank criteria [25] have participated. Ages ranged from 59 to 77 years, and patients were in a mild or moderate stage of the disease (H\&Y greater than or equal to 2.5 in their ON state). The average age was 67.6 years, and the average H\&Y in OFF state was 3.04. Before the experiments, all patients were given verbal explanations and had signed informed consent forms. The experimental protocol was approved by the local Ethics Review Committee.

Signals employed in this work were gathered using the 9x2 inertial measurement unit [27]. This sensor unit was located on the waist near the iliac crest by using a hypoallergenic neoprene belt, as shown in Figure 1. 9x2 is a wearable device for research purposes designed at the CETpD that has been used in other studies with Parkinson's disease patients [19]. It includes a triaxial accelerometer, a Bluetooth communication module, a memory storage unit and a Li-ion rechargeable battery. Its size is $99 \times 52 \times 19 \mathrm{~mm}^{3}$, and its weight is $83 \mathrm{~g}$. (including battery). Signals were captured by the 9x2 device at $200 \mathrm{~Hz}$ and saved in a microSD card. These signals were subsampled to $f_{s}=40 \mathrm{~Hz}$ to apply the methods, as described in the previous section. The device's accelerometer full scale was set to $\pm 6 \mathrm{G}$ range [27].

A portable video camera was used to record patients while they were performing the experimental 
procedure, as described in the following subsections.

\subsection{Experimental procedure}

With the aim of collecting data as real as possible, signals were collected at patients' home. This fact has shown to be important, since PD symptoms may vary depending on the environment: the freezingof-gait frequency of appearance is reduced in the laboratory setting [28]. The experiment was also designed with the aim of gathering signals both with and without bradykinesia for each patient. With this purpose, a widely extended method in clinical experiments was used. A state in which PD symptoms are evident was elicited by either removing or reducing the patients' antiparkinsonian medication prior to the experiment, with the aim of forcing a low dopamine-level. Afterwards, patients performed a series of tests consisting of several activities that, although they were scripted, their execution was free. These activities included, among others, walking around their home and showing it to the researchers, carrying a full glass of water from the kitchen to another room, a freezing-of-gait provocation test and walking for approximately $10 \mathrm{~m}$. in a straight line. Between activities, patients sat several times in a chair, so many postural transitions were included in the recordings. During the data gathering, other non-scripted activities took place, such as answering a phone call or other unexpected situations. This data collection took from 10 to 30 minutes. Once the patient completed these activities, regular antiparkinsonian medication was taken, and researchers waited for the patient to recover a good motor control. Then, the patient proceeded to perform the same tests again.

Prior to each data collection, clinicians administered the motor part of the UPDRS scale. Given that 12 patients were recruited and each patient participated twice in the data collection, a total of 24 UPDRS were obtained, as well as 24 sets of signals. All data were collected by research assistants who had been specifically trained in the study procedures and administration of the corresponding questionnaires, on the basis of specific guidelines.

The gold standard for bradykinesia detection was generated from the video recordings taken during each data collection session while patients were performing the activities and they wore the sensor. Video recordings were synchronised with the accelerometer signals and, then, they were labelled by trained experts. In regards to this paper, labels consisted on the presence or absence of bradykinetic gait, for each time patients walked, which were then extrapolated to the signals. 
Note that bradykinesia severity is very complex to quantify during activities of daily living because of the difficulty in distinguishing voluntary against pathological movements. For this reason, only the presence and absence of bradykinesia has been labelled on the videos, while the severity is assessed by specific items of the UPDRS, which are described in subsection 4.4.

\subsection{Data analysis for bradykinesia detection and adjustment of $\beta$}

The approach presented in Section 3 provides an output for every walking bout by averaging the strides in it, i.e. $\bar{m}_{n}=\sum_{i=3}^{k_{n}-2} \frac{m_{i}^{n}}{k_{n}-4}$. This output is compared with $\beta$ according to Equation (3) in order to estimate the presence of bradykinesia. The way in which $\beta$ is obtained is explained below in this same subsection.

Bradykinesia estimations are contrasted with the gold-standard given by clinicians, which is based on the video recordings. This gold-standard represents whether each walking bout is bradykinetic or not, so for every $\bar{m}_{n}$ there is its corresponding ground-truth $v_{n}$.

Data available for each patient consists of the set of walking-bout fluidity values and their goldstandard $\left\{\left(\bar{m}_{n}, v_{n}\right)\right\}_{i=1 \ldots 12}$, the vector-representation of each patient $\{\boldsymbol{p}\}_{i=1 \ldots 12}$ (see Equation 4 ), and their optimal threshold values $\{\hat{\beta}\}_{i=1 \ldots 12}, \hat{\beta}_{i} \in \mathbb{R}$. From them, mapping $h$ (defined in Section 3.3) is implemented through a leave-one-patient-out method. This mapping $h$ provides the $\beta$ value for a patient, which is used in Equation 3 to predict whether his/her walking bouts are bradykinetic or not. More specifically, the $n^{\text {th }}$ walking bout is predicted as bradykinetic if $\bar{m}_{n} \leq \beta$, and as not bradykinetic otherwise. From the predicted outputs of a patient and the corresponding ground-truths, accuracy, specificity and sensitivity values are obtained as follows. Accuracy is the rate of walking bouts that are correctly identified, either as bradykinetic or non-bradykinetic. Specificity is the rate of non-bradykinetic bouts that are successfully predicted, while sensitivity is the rate of correctly identified bradykinetic walking bouts.

Optimal thresholds $\hat{\beta}_{i}$ were found for each patient based on the corresponding labelled dataset $\left\{\left(\bar{m}_{n}, v_{n}\right)\right\}_{i}$. The interval $\left[\min \left(\left\{\bar{m}_{n}\right\}_{i}\right), \max \left(\left\{\bar{m}_{n}\right\}_{i}\right)\right]$ was sampled at steps of 0.1 , so that the set of values $\Gamma_{\mathrm{i}}=\left\{\min \left(\left\{\bar{m}_{n}\right\}_{i}\right), \min \left(\left\{\bar{m}_{n}\right\}_{i}\right)+0.1, \min \left(\left\{\bar{m}_{n}\right\}_{i}\right)+0.2, \ldots, \max \left(\left\{\bar{m}_{n}\right\}_{i}\right)\right\}$ was obtained. Each element from $\Gamma_{\mathrm{i}}$ was tested to classify patient's labelled dataset $\left\{\left(\bar{m}_{n}, v_{n}\right)\right\}_{i}$ by using Equation (3), giving a specificity and a sensitivity value for each tested value. Finally, $\hat{\beta}_{i}$ was set as the element from $\Gamma_{i}$ that maximised the geometric mean between its specificity and sensitivity. 
Thresholds $\beta_{i}$ used in Equation (5) were obtained by means of a leave-one-patient out procedure. To this end, the training dataset for patient $i$ consists of, on the one hand, optimal thresholds $\hat{\beta}_{1}, \ldots, \hat{\beta}_{i-1}, \hat{\beta}_{i+1}, \hat{\beta}_{12}$ and, on the other hand, vectorised patients' information $\boldsymbol{p}_{1}, \ldots, \boldsymbol{p}_{i-1}, \boldsymbol{p}_{i+1}, \boldsymbol{p}_{12}$. This training dataset for patient $i$ was used to train the $\epsilon$-SVR model that approximates $h$, as described in Equation (5). Through these patterns, a 10-fold cross-validation procedure was used to obtain $C, \gamma$, and $\epsilon$ optimal values, by selecting those that minimised the Root Mean Squared Error (RMSE). Tested values for $C$ and $\gamma$ were $10^{-3}, 10^{-2}, \ldots, 10^{2}, 10^{3}$, and those tested for $\epsilon$ were $2^{1}, 2^{0}, \ldots, 2^{-10}$. Once the optimal values were found, the final regression model was obtained through these values and the training dataset for patient $i$. Note that there were as many regression models as patients, since a leave-one-patientout was used. Finally, threshold $\beta_{i}$ was set as the output of the regression model for $\boldsymbol{p}_{i}$.

This method for estimating thresholds $\beta_{i}$ has the advantage of not requiring any label from patient $i$. Data used are other patients' optimal thresholds $\hat{\beta}$ and the vector representation $\boldsymbol{p}$ of all patients (included the testing patient). As Equation (4) shows, vector $\boldsymbol{p}_{i}$ only requires the testing patient to walk with the sensor (without having to label any walking bout) and to know the age and H\&Y of the patient.

\subsection{Data analysis for bradykinesia severity estimation as a regression of UPDRS scores}

The main objective of this estimation is to automatically quantify the bradykinesia severity in clinical terms so that health professionals can easily understand the provided information. To this end, UPDRS scores were approximated based on the fluidity measurements, according to mapping $z$ in Equation (6). However, in contrast with walking-bout labels presented in subsection 4.3, UPDRS scores were obtained once for each data collection session (each patient with and without medication). This way, UPDRS was administered twice to each patient.

The analysed UPDRS items are those that are more closely related to bradykinesia and gait, which are: gait (item 22) and bradykinesia, and hypokinesia (item 24). In addition to this, UPDRS has been analysed in order to cluster its items and identify which different aspects of the disease are covered with the scale [29]. As a result, different factors were identified, such as factor I, which includes the axial function, balance and gait items. More specifically, it comprises items 10 and 11 (right and left lower extremity rigidity) and items 20 to 24 (arising from a chair, posture, gait, postural stability and body bradykinesia and hypokinesia). Therefore, the UPDRS factor that covers bradykinesia assessment is 
factor I, and it is included in the analysis. This way, mapping $z$ presented in Equation (6) is found for this three scores: item 22, item 24 and UPDRS Factor I.

The relation among bradykinesia measurements $m_{i}^{n}$ and symptom's severity quantified by UPDRS items was examined in two different ways. On the one hand, linear relation was evaluated based on correlation coefficients. On the other hand, bradykinesia severity was measured by evaluating the ability to estimate the UPDRS item scores through a regression model.

First, a correlation analysis was done to confirm linear relation. Pearson correlation coefficients were obtained for averaged bradykinesia measurements from each data collection session, and the score of the specific UPDRS items from each session.

Second, UPDRS scores were estimated according to the mapping $z$ from Equation (6) implemented through an $\epsilon-$ SVR. This mapping was implemented similarly to mapping $h$ presented in the previous section. In this case, data available from each patient consisted of the UPDRS ratings for items 22, 24 and Factor $I\left\{u_{22}^{w}, u_{22}^{\bar{w}}, u_{24}^{w}, u_{24}^{\bar{w}}, u_{F I}^{w}, u_{F I}^{\bar{w}}\right\}_{i}$ (obtained with and without medication, $w$ and $\bar{w}$, respectively); and the vector representation of each patient, which was obtained twice, represented by $\left\{\boldsymbol{p}_{i}^{w}, \boldsymbol{p}_{i}^{\bar{w}}\right\}_{i}$. Vectors $\boldsymbol{p}_{i}^{w}$ and $\boldsymbol{p}_{i}^{\bar{w}}$ contain the H\&Y and age of patient $i$, and the statistics of the fluidity measurements from each data collection session.

Bradykinesia severity measured by UPDRS is estimated through the following leave-one-patient-out approach. When evaluating patient $i$ and a specific UPDRS item, the training dataset was composed of the UPDRS values and the vector representation from the remaining 11 patients (see Eq. 4). Given this dataset, a 10-fold cross-validation procedure was used to obtain $C, \gamma$, and $\epsilon$ optimal values, by selecting those values that minimised the RMSE. Tested values for $C$ and $\gamma$ were $10^{-3}, 10^{-2}, \ldots, 10^{2}, 10^{3}$, and those tested for $\epsilon$ were the range of the UPDRS item divided by $2^{0}, 2^{1}, \ldots, 2^{10}$. Once the optimal values were found, the final regression model was obtained through these values and the training dataset for patient $i$. Note that there were as many regression models obtained as patients and UPDRS items $(12 \cdot 3=36$ models). Finally, estimated UPDRS scores were the output of the regression model for $\boldsymbol{p}_{i}^{w}$ and $\boldsymbol{p}_{i}^{\bar{w}}$. This method presents the same advantages than the bradykinesia detection method described in the previous subsection, since it does not require labelled data from the testing patient. 


\section{Results}

\subsection{Bradykinesia detection}

Bradykinesia detection results are presented in Table 1. Each row reports the accuracy, specificity, and sensitivity obtained for each patient following the leave-one-patient-out approach described. Each row also includes the total number of patterns (i.e. walking bouts) in each class (bradykinetic and non-bradykinetic). The average specificity and sensitivity in detecting bradykinetic gait is $89.07 \%$ and $92.52 \%$, respectively, while the average accuracy is $91.81 \%$. This table also presents the optimal thresholds $\hat{\beta}$ and the estimated $\beta$ found for each patient.

Bradykinesia detection algorithm shows excellent results with an average accuracy higher than $90 \%$. Individually, the lowest accuracy values are above $85 \%$ suggesting, thus, a good reliability of the method. Despite the high accuracy values, it is observed that patient 11 presents a rather low specificity (66\%). However, this value is due to the low number of non-bradykinetic patterns (3 patterns).

In addition, Table 1 shows that several patterns were employed (average of 29.58 per patient), each one corresponding to a walking bout. This way, gait assessment provided a frequent estimation of the motor state of the patient.

Finally, the estimation of $\beta$ through the leave-one-patient-out regression shows that their values are accurately approximated. The maximum difference between $\beta$ and $\hat{\beta}$ is approximately $0.5(\sim 7 \%)$ and the average error in the estimation of $\beta$ is -0.02 with a standard deviation of 0.25 . The average percentage of the absolute value of the error is $3.4 \%$. In consequence, it is considered that the obtained threshold estimation is reliable.

\begin{tabular}{cccccccc}
\hline Patient & Accuracy & Sensitivity & Specificity & $\begin{array}{c}\text { Brad. } \\
\text { patterns }\end{array}$ & $\begin{array}{c}\text { Non-brad. } \\
\text { patterns }\end{array}$ & $\hat{\beta}$ & $\beta$ \\
\hline 1 & $85.42 \%$ & $90.00 \%$ & $77.78 \%$ & 30 & 28 & 6.77 & 6.59 \\
2 & $94.59 \%$ & $96.15 \%$ & $90.91 \%$ & 26 & 11 & 7.34 & 7.62 \\
3 & $87.50 \%$ & $86.67 \%$ & $88.89 \%$ & 15 & 9 & 7.56 & 8.09 \\
4 & $96.00 \%$ & $100.00 \%$ & $91.67 \%$ & 13 & 12 & 5.10 & 4.76 \\
5 & $91.30 \%$ & $94.12 \%$ & $83.33 \%$ & 17 & 6 & 5.12 & 5.43 \\
6 & $86.67 \%$ & $90.48 \%$ & $77.78 \%$ & 21 & 9 & 4.89 & 4.75 \\
7 & $100.00 \%$ & $100.00 \%$ & $100.00 \%$ & 7 & 7 & 6.13 & 6.27
\end{tabular}




\begin{tabular}{cccccccc}
8 & $92.59 \%$ & $88.24 \%$ & $100.00 \%$ & 17 & 10 & 6.52 & 6.57 \\
9 & $100.00 \%$ & $100.00 \%$ & $100.00 \%$ & 13 & 8 & 5.66 & 5.66 \\
10 & $85.71 \%$ & $89.47 \%$ & $77.78 \%$ & 19 & 9 & 5.34 & 5.46 \\
11 & $88.00 \%$ & $89.36 \%$ & $66.67 \%$ & 47 & 3 & 5.11 & 4.91 \\
12 & $89.29 \%$ & $83.33 \%$ & $100.00 \%$ & 18 & 10 & 7.74 & 7.70 \\
\hline Average & $\mathbf{9 1 . 8 1 \%}$ & $\mathbf{9 2 . 5 2} \%$ & $\mathbf{8 9 . 0 7 \%}$ & $\mathbf{2 0 . 2 5}$ & $\mathbf{9 . 3 3}$ & $\mathbf{6 . 1 1}$ & $\mathbf{6 . 1 5}$ \\
\hline
\end{tabular}

Table 1. Results on bradykinetic gait detection. Each classified pattern corresponds to a walking bout with 5 or more strides. Optimal threshold $\hat{\beta}$ and the estimated threshold $\beta$ are presented in the last two columns.

\subsection{Bradykinesia severity estimation}

A linear relation between the average bradykinesia fluidity value and the different UPDRS items has been observed, which is shown in Table 2, in which Pearson correlation coefficients and associated $p$ values are presented. All correlation coefficients are statistically significant $(p<0.001)$ and negative since higher fluency values are related to lower UPDRS values. Gait score (UPDRS Item 22) provides an excellent correlation $(r=-0.91)$ and Bradykinesia and Hypokinesia score (item 24), and UPDRS Factor I achieve high values $(-0.90<r<-0.80)$.

Table 3 presents the results on estimating bradykinesia severity based on the same UPDRS items used in Table 2. The estimation is performed based on the $\varepsilon$-SVR model trained through a leave-onepatient-out approach, as described in Section 4. Average errors are presented through the mean and standard deviation error, the RMSE and the normalised RMSE (NRMSE). The item that is most accurately estimated is Item 22 (gait) with NRMSE $=7.8 \%$. Items 24 and UPDRS Factor I are estimated with $14.75 \%$ and $10 \%$ of NRMSE, respectively. The mean error of Item 22, Item 24 and Factor I with respect to the measurement range is $1 \%, 2.5 \%$ and $3 \%$, respectively.

\begin{tabular}{cccc}
\hline Description & $\begin{array}{c}\text { UPDRS Item 22 } \\
\text { (gait) }\end{array}$ & $\begin{array}{c}\text { UPDRS Item 24 } \\
\text { (bradyk. and hypok.) }\end{array}$ & UPDRS Factor I \\
\hline $\begin{array}{c}\text { Correlation between UPDRS scores } \\
\text { and absolute fluidity values }\end{array}$ & $r=-0.912$ & $r=-0.808$ & $r=-0.834$ \\
& $p<0.001$ & $p<0.001$ & $p<0.001$ \\
\hline
\end{tabular}

Table 2. Correlation coefficients and p-values between bradykinesia-related UPDRS scores and the fluidity measurements obtained by the sensor. 


\begin{tabular}{ccccc}
\hline Regression variable & Range & $\begin{array}{c}\text { Mean error } \\
\text { (std. deviation) }\end{array}$ & RMSE & NRMSE \\
\hline UPDRS item 22 (gait) & {$[0-4]$} & $-0.04(0.31)$ & 0.31 & $7.77 \%$ \\
UPDRS item 24 (bradyk. and hypok.) & {$[0-4]$} & $0.10(0.59)$ & 0.59 & $14.75 \%$ \\
UPDRS Factor I & {$[0-28]$} & $0.84(3.04)$ & 3.09 & $11.03 \%$ \\
\hline
\end{tabular}

Table 3. UPDRS estimation results based on motion fluency values and $\varepsilon-S V R$. The range of the UPDRS score is included. Mean and standard deviation error among the 12 PD patients is reported, and their corresponding Root Mean Squared Error (RMSE) and Normalised RMSE (NRMSE).

Results presented in Table 3 show that the best estimated UPDRS is UPDRS Item 22. This item provides the lowest error since it rates gait, which is the main parameter examined by the waist-worn sensor. Bradykinesia and hypokinesia ratings (item 24$)$ are estimated through a higher error (14\% compared to $7 \%)$. Two factors may influence this lower accuracy. Firstly, item 24 not only rates gait but also evaluates overall movement, such as arm-swing and "poverty of movement in general". In consequence, given that the sensor is examining gait, item 22 naturally matches with the fluidity measurements and item 24 introduces some movement aspects that are not evaluated. Secondly, item 24 is more subjective than item 22. On the one hand, item 24 (bradykinesia) rates "Body bradykinesia and hypokinesia (combining slowness, hesitancy, decreased arm-swing, small amplitude, and poverty of movement in general)", distinguishing among the following degrees: "Minimal", "Mild degree", and "Moderate". On the other hand, item 22 (gait) rates "Gait" distinguishing "Walks slows, short steps", "Walks with difficulty, requires assistance, may have festination", and "Severe disturbance requiring assistance". Thus, the definition of item 22 is more specific than item 24, since distinguishing between moderate and mild slowness is subjective, in contrast to determining if a patient requires assistance to walk. In consequence, subjectivity may have introduced variations into item 24 that cannot be determined by the sensor and they may be reflected as an increased error.

Regarding UPDRS Factor I, the results also show a higher error than item 22, although lower than item 24. The higher error is considered to come from the different assessments included in factor I (rigidity in items 10-11, posture transition in item 20, posture in item 21, gait in item 22, postural stability in item 23 and bradykinesia in item 24). However, the good correlation and the rather low error show a direct relation between the sensor fluidity measurements and this factor. Compared to item 24, factor I 
includes many items that are not as subjective as this item; such as item 23, which clearly rates the response to a sudden strong posterior displacement; item 20, that describes with detail how to mark arising from a chair; and item 22. This way, Factor-I reduced subjectivity may have decreased the variability in the observations and improved the regression model that estimates its values.

\section{Discussion}

Compared to previous works, our method presents higher correlations between UPDRS scores and specific values extracted from inertial signals. In the previous work of Salarian et al. [8], they obtained correlations between -0.42 and -0.76 by using a wrist sensor. On the other hand, Zwartjes et al. [9] obtained correlations up to -0.70 by using 4 sensors; although none of the parameters that they extracted was significantly correlated with the UPDRS scores that represent bradykinesia and hypokinesia. In our case, all correlations are significant, and coefficients up to 0.91 are achieved by using a waist sensor. These works did not report specificity and sensitivities on detecting UPDRS scores nor bradykinetic periods.

Regarding bradykinesia severity estimation, Cancela et al. [10] reported the results of detecting UPDRS bradykinesia scores with an average accuracy of $74.4 \%$ by using a set of 5 sensors. The results we have obtained in the present work, however, consist in $85.3 \%$ of average accuracy (14.75\% NRMSE according to Table 3), which are $10 \%$ higher. In addition to this, Cancela et al. used 5 sensors, in contrast to the single sensor employed by our method.

Patel et al. [5] estimated different UPDRS items by using a set of 8 accelerometers. UPDRS ratings were given while patients performed specific exercises, such as finger-to-nose, finger tapping, heel tapping, etc. More specifically, they estimated bradykinesia-related items with an average error of $1.7 \%$. However, it requires patients to do specific motion tasks, such as finger tapping, and to wear the set of 8 sensors. In consequence, the approach presented by Patel et al. cannot assess bradykinesia in daily life activities, in contrast to the approach presented in this work, which only requires patients to walk.

The main limitation of the method presented in this work consists in its dependence on gait analysis, i.e. in order to estimate the presence and severity of bradykinesia, patients have to walk (at least 5 strides to determine the presence of bradykinesia). Patients included in this study had H\&Y in ON of 2.5 or lower, although the maximum H\&Y value in OFF was of 4 (i.e. all patients were able to walk unassisted). Hence, patients who are unable to walk $(H \& Y=5)$ would not benefit from the monitoring of the presented 
method. However, the majority of patients has a lower H\&Y; in the case of a previous study with 1768 PD patients [30], when the disease duration was 6 to 10 years, only $3.5 \%$ of patients had a H\&Y=5.

The use in the clinical practice of a wearable sensor implementing the proposed method may be feasible under some circumstances. More concretely, the sensor requires patients to walk 5 strides or more for several times (at least 7 walking bouts, according to Table 1) in a time interval of 10 to 30 minutes, according to the data collection section. Provided that these conditions are satisfied, patients wearing the sensor over few days could benefit from the monitoring of the bradykinesia severity several times a day. As a result, the information collected would consist in an objective measurement of motor fluctuations in Parkinson's disease that clinicians may use to change or improve their patients' medication regime.

The approach presented in this paper not only outperforms the results previously reported in the literature but also presents the advantage of employing a single accelerometer located in the waist instead of several sensors, which facilitates its usage to PD patients, who have motor limitations and for who too complex systems may result extremely cumbersome. Although more extensive validation is needed, results show that an accurate assessment can be obtained from a single waist-worn sensor.

\section{Conclusions}

This paper presents a new method to objectively detect and automatically assess bradykinesia in PD patients by means of a waist-worn triaxial accelerometer. This method exploits the signals acquired by the sensor and unobtrusively analyses gait during ADL. The method has been evaluated in 12 PD patients at their home while doing several ADL, and in two different states: with and without medication (ON and OFF states).

Three main contributions are drawn from this paper. Firstly, the characterisation of strides enables a reliable detection of bradykinetic periods (average accuracy higher than 90\%). Secondly, the same characterisation of strides is shown to be correlated with specific UPDRS items, being the $22^{\text {nd }}$ item (gait) the most correlated one $(r=-0.91)$, and having a high correlation with the $24^{\text {th }}$ item (bradykinesia and hypokinesia) with $r=-0.80$. Thirdly, these high correlations translate into errors of approximately $10 \%$ when estimating the UPDRS scores through an $\varepsilon$-SVR model. Compared to previous works [8] [9], our approach provides higher correlations with UPDRS. Regarding the estimation of the UPDRS items [10], 
only the work of Patel et al. [5] outperforms our accuracy; however, their approach requires the execution of specific motion tasks such as finger and heel tapping. In addition to this, most of previous works employ several sensors.

This way, the main advantages of our proposed approach relies on, first of all, its simplicity, since a single waist-worn accelerometer is needed. Additionally, the presented method is capable of monitoring bradykinesia without specific motion exercises and with high accuracies. Furthermore, it is a patientindependent approach, in the sense that it does not require labelled data in order to be used with a new PD patient. More specifically, the method needs the H\&Y and the age of the new patient, and several walking bouts that could be obtained during a day (without labelling them). Based on these data, the method is personalised to the new patient through regression models and, then, bradykinesia is detected and rated. The only limitation is that patients are required to walk; nonetheless, it is a frequent activity commonly done during ADL.

The approach presented in this paper supports current trends on continuous monitoring of Parkinson's disease. In this case, bradykinetic periods may be reported with a single waist-worn sensor. Its clinical relevance relies on the fact that bradykinesia is the symptom that best correlates with dopaminergic deficiency. Thus, by knowing bradykinesia fluctuations, neurologists could then tailor the medication regimen to avoid OFF states and, in the long term, accurately follow the disease evolution. Additionally, clinical studies may take advantage of this kind of systems in order to objectively measure the clinical effect of new therapies. Finally, real-time monitoring provided by a single waist-worn sensor may be employed to control the doses administered by current infusion pumps used in advanced PD in order to reduce medication side effects and OFF periods.

\section{Acknowledgements}

This data collection has been performed within the framework of the MoMoPa2 Project (Monitoring the mobility of Parkinson's patients for therapeutic purposes 2 - PI12/03028) funded by the Instituto de Salud Carlos III - Ministerio de Economía y Competividad and the European Regional Development Fund (ERDF).

This study was also conducted within the Monitoring the Mobility of Parkinson's Patients for Therapeutic Purposes Project (DTS15/00209), funded by the Instituto de Salud Carlos III - Ministerio de Economía, Industria y Competitividad - and the European Regional Development Fund. 


\section{Author Contributions}

Albert Samà and Carlos Pérez-López have performed the design of the method as well as written the paper. Daniel Rodríguez-Martín has performed the hardware of the system and he has written and reviewed the paper. Alejandro Rodríguez-Molinero, Andreu Català, Joan Cabestany, and Joan Manuel Moreno-Arostegui have participated in the conception and design of the study and have reviewed the paper. Eva de Mingo and Alejandro Rodríguez-Molinero have carried out the data collection, medical issues and they have reviewed the paper.

\section{Conflicts of Interest}

Alejandro Rodríguez-Molinero, Albert Samà, Carlos Pérez-López, Andreu Català, Juan Manuel Moreno and Joan Cabestany are shareholders of Sense4Care, which is a spin-off company of Universitat Politècnica de Catalunya that may commercialise the results of this research device in a near future. These authors declare that the possible commercialization of the product is a research outcome, not being the design, the analysis, the interpretation of the results or the conclusions affected by commercial interests

\section{References}

[1] J. Jankovic, Parkinson's disease: clinical features and diagnosis, J. Neurol. Neurosurg. Psychiatry . 79 (2008) 368-376. doi:10.1136/jnnp.2007.131045.

[2] A. Berardelli, J.C. Rothwell, P.D. Thompson, M. Hallett, Pathophysiology of bradykinesia in Parkinson's disease, Brain. 124 (2001) 2131-2146. http://www.ncbi.nlm.nih.gov/pubmed/11673316.

[3] F.J.G. Vingerhoets, M. Schulzer, D.B. Calne, B.J. Snow, Which clinical sign of Parkinson's disease best reflects the nigrostriatal lesion?, Ann. Neurol. 41 (1997) 58-64. doi:10.1002/ana.410410111.

[4] D.A. Heldman, T. Mera, A. Espay, M. Payne, J. Giuffrida, Quantitative Home-Based Monitoring of Parkinson's Disease Motor Symptoms, 2011.

[5] S. Patel, K. Lorincz, R. Hughes, N. Huggins, J. Growdon, D. Standaert, et al., Monitoring Motor Fluctuations in Patients With Parkinson' s Disease Using Wearable Sensors, IEEE Trans. Inf. Technol. Biomed. 13 (2009) 864-873.

[6] R.J.W. Dunnewold, J.I. Hoff, H.C.J. van Pelt, P.Q. Fredrikze, E.A.H. Wagemans, B.J.J. van Hilten, Ambulatory Quantitative Assessment of Body Position, Bradykinesia, and Hypokinesia in Parkinson's Disease, J. Clin. Neurophysiol. 15 (1998) 235-242.

[7] A. Salarian, Ambulatory monitoring of motor functions in patients with parkinson's disease using kinematic sensors, École polytechnique federale de Lausanne, 2006.

[8] A. Salarian, H. Russmann, C. Wider, P.R. Burkhard, F.J.G. Vingerhoets, K. Aminian, Quantification of tremor and bradykinesia in Parkinson's disease using a novel ambulatory monitoring system., IEEE Trans. Biomed. Eng. 54 (2007) 313-322. doi:10.1109/TBME.2006.886670.

[9] D. Zwartjes, T. Heida, J. van Vugt, J. Geelen, P. Veltink, Ambulatory Monitoring of Activities and Motor Symptoms in Parkinson's Disease., IEEE Trans. Biomed. Eng. 57 (2010) 2778-2786. doi:10.1109/TBME.2010.2049573. 
[10] J. Cancela, M. Pansera, M.T. Arredondo, J.J. Estrada, M. Pastorino, L. Pastor-Sanz, et al., A comprehensive motor symptom monitoring and management system: The bradykinesia case, in: Eng. Med. Biol. Soc. (EMBC), 2010 Annu. Int. Conf. IEEE, 2010: pp. 1008-1011. doi:10.1109/IEMBS.2010.5627775.

[11] M. Pastorino, J. Cancela, M.T. Arredondo, M. Pansera, L. Pastor-Sanz, F. Villagra, et al., Assessment of bradykinesia in Parkinson's disease patients through a multi-parametric system, in: Eng. Med. Biol. Soc. EMBC, 2011 Annu. Int. Conf. IEEE, 2011: pp. 1810-1813. doi:10.1109/IEMBS.2011.6090516.

[12] R.I. Griffiths, K. Kotschet, S. Arfon, Z.M. Xu, W. Johnson, J. Drago, et al., Automated assessment of bradykinesia and dyskinesia in Parkinson's disease, J. Parkinsons. Dis. 2 (2012) 47-55. doi:10.3233/JPD-201211071.

[13] J.-W. Kim, J.-H. Lee, Y. Kwon, C.-S. Kim, G.-M. Eom, S.-B. Koh, et al., Quantification of bradykinesia during clinical finger taps using a gyrosensor in patients with Parkinson's disease, Med. Biol. Eng. Comput. 49 (2011) 365-371.

[14] H. Dai, H. Lin, T.C. Lueth, Quantitative assessment of parkinsonian bradykinesia based on an inertial measurement unit, Biomed. Eng. Online. 14 (2015) 68. doi:10.1186/s12938-015-0067-8.

[15] T.O. Mera, D.A. Heldman, A.J. Espay, M. Payne, J.P. Giuffrida, Feasibility of home-based automated Parkinson's disease motor assessment., J. Neurosci. Methods. $203 \quad$ (2012) 152-6. doi:10.1016/j.jneumeth.2011.09.019.

[16] L. Rochester, S.F.M. Chastin, S. Lord, K. Baker, D.J. Burn, Understanding the impact of deep brain stimulation on ambulatory activity in advanced Parkinson's disease, J. Neurol. 259 (2012) 1081-1086.

[17] J.T. Cavanaugh, T.D. Ellis, G.M. Earhart, M.P. Ford, K.B. Foreman, L.E. Dibble, Capturing Ambulatory Activity Decline in Parkinson Disease, J. Neurol. Phys. Ther. JNPT. 36 (2012) 51.

[18] C.C. Yang, Y.L. Hsu, A review of accelerometry-based wearable motion detectors for physical activity monitoring., Sensors (Basel). 10 (2010) 7772-7788. doi:10.3390/s100807772.

[19] A. Samà, C. Perez-Lopez, J. Romagosa, D. Rodriguez-Martin, A. Catala, J. Cabestany, et al., Dyskinesia and motor state detection in Parkinson's Disease patients with a single movement sensor, in: D. Haemmerich, D. Panescu, J. Pearce, P. Prakash, E. Sloane, P. Soda, et al. (Eds.), Eng. Med. Biol. Soc. 2012. 34th Annu. Int. Conf. IEEE, San Diego, 2012: pp. 1194-1197. doi:10.1109/EMBC.2012.6346150.

[20] A. Rodríguez-Molinero, A. Samà, D.A. Pérez-Martínez, C. Pérez López, J. Romagosa, À. Bayés, et al., Validation of a Portable Device for Mapping Motor and Gait Disturbances in Parkinson's Disease, JMIR mHealth uHealth. 3 (2015) e9. doi:10.2196/mhealth.3321.

[21] E. Chu, A. George, Inside the FFT black box. Serial and Parallel Fast Fourier Trnasform Algorithms, CRC Press LLC, Boca Raton, 2000.

[22] H. Nyquist, Certain topics in telegraph transmission theory, Am. Inst. Electr. Eng. Trans. 47 (1928) 617-644.

[23] W. Zijlstra, A.L. Hof, Assessment of spatio-temporal gait parameters from trunk accelerations during human walking, Eur. J. Appl. Physiol. 18 (2003) 39-44. doi:10.1016/S0966-6362(02)00190-X.

[24] W. Zijlstra, A.L. Hof, Assessment of spatio-temporal parameters during unconstrained walking, Eur. J. Appl. Physiol. 92 (2004) 39-44. doi:10.1016/S0966-6362(02)00190-X.

[25] V.N. Vapnik, The Nature of Statistical Learning Theory, second, Springer-Verlag, New York, 2013.

[26] A.J. Smola, B. Schölkopf, A tutorial on support vector regression, Stat. Comput. 14 (n.d.) 199-222. doi:10.1023/B:STCO.0000035301.49549.88. 
[27] D. Rodríguez-Martín, C. Pérez-López, A. Samà, J. Cabestany, A. Català, A Wearable Inertial Measurement Unit for Long-Term Monitoring in the Dependency Care Area, Sensors. 13 (2013) 14079-14104. doi:10.3390/s131014079.

[28] A. Nieuwboer, W. de Weerdt, R. Dom, E. Lesaffre, A frequency and correlation analysis of motor deficits in Parkinson patients, Disabil. Rehabil. 20 (1998) 142-150.

[29] G.T. Stebbins, C.G. Goetz, A.E. Lang, E. Cubo, Factor analysis of the motor section of the unified Parkinson's disease rating scale during the off-state, Mov. Disord. 14 (1999) 585-589. doi:10.1002/15318257(199907)14:4<585::AID-MDS1006>3.0.CO;2-3.

[30] K. Sato, T. Hatano, K. Yamashiro, M. Kagohashi, K. Nishioka, N. Izawa, et al., Prognosis of Parkinson's disease: Time to stage III, IV, V and to motor fluctuations, Mov. Disord. 21 (2006) 1384-1395. doi:10.1002/mds.20993. 